\title{
Association between vancomycin pharmacokinetic/pharmacodynamic parameters, patient characteristics, and mortality in patients with bacteremia caused by vancomycin-susceptible Enterococcus faecium: a single-center retrospective study
}

Ichiro Nakakura*iD, Kota Sakakura, Kaori Imanishi, Rumi Sako and Kunio Yamazaki

\begin{abstract}
Background: Vancomycin is commonly used to treat Enterococcus faecium (E. faecium) bacteremia. However, there are very few studies on the association between the trough concentration, area under the curve from 0 to $24 \mathrm{~h}$ /minimum inhibitory concentration $\left(\mathrm{AUC}_{24} / \mathrm{MIC}\right.$ ) ratio, and the therapeutic effect of vancomycin on $E$. faecium bacteremia. This study aimed to investigate the associations between vancomycin pharmacokinetic/ pharmacodynamic parameters, patient characteristics, and mortality in patients with E. faecium bacteremia.

Methods: This retrospective study included patients with E. faecium bacteremia who received vancomycin between April 2012 and February 2018 at a single acute care hospital in Japan. Patients who received renal replacement therapy (hemodialysis or continuous hemodiafiltration), had an unmeasured serum vancomycin concentration, with unmeasured laboratory values, or received other antibiotics for treating $E$. faecium bacteremia were excluded from the study. The bivariate associations between 30-day all-cause mortality and patient characteristics were assessed.

Results: Among 87 patients diagnosed with E. faecium bacteremia, 45 were included in the final analysis. Of these, 12 (26.7\%) died within 30 days of the diagnosis. The vancomycin trough concentration was higher in the 30-day all-cause mortality patients than in the survival patients ( $20.5 \mathrm{vs} .14 .6 \mu \mathrm{g} / \mathrm{mL} ; P=0.022)$. There was no significant difference in the proportion of patients with a vancomycin $\mathrm{AUC}_{24} / \mathrm{MIC} \leq 389$ between the groups. The 30-day all-cause mortality patients showed a higher Charlson Comorbidity Index (CCl) and Sequential Organ Failure Assessment score at the first measurement of the vancomycin trough concentration than the survival patients. The same finding was observed among patients with a high CCI score ( $\geq 5$ points).
\end{abstract}

Conclusions: Whereas the vancomycin trough concentration and $\mathrm{AUC}_{24} / \mathrm{MIC}$ ratio were not associated with mortality in patients with E. faecium bacteremia, disease severity was associated with mortality in these patients.

Keywords: Vancomycin, Pharmacokinetics, Pharmacodynamics, Enterococcus faecium, Bacteremia, Prognosis, Mortality

\footnotetext{
* Correspondence: xxxichiroxxx.0421@gmail.com

Department of Pharmacy, National Hospital Organization Osaka National

Hospital, 2-1-14 Hoenzaka, Chuo-ku, Osaka 540-0006, Japan
}

(c) The Author(s). 2019 Open Access This article is distributed under the terms of the Creative Commons Attribution 4.0 International License (http://creativecommons.org/licenses/by/4.0/), which permits unrestricted use, distribution, and reproduction in any medium, provided you give appropriate credit to the original author(s) and the source, provide a link to the Creative Commons license, and indicate if changes were made. The Creative Commons Public Domain Dedication waiver (http://creativecommons.org/publicdomain/zero/1.0/) applies to the data made available in this article, unless otherwise stated. 


\section{Background}

Enterococcus faecium (E. faecium) is commonly found among the commensal microflora in the human gut and mainly causes urinary tract infections, wound infections, endocarditis, and bacteremia [1]. Vancomycin-resistant E. faecium is a frequent isolate in the USA [2] but an infrequent one in Japan and Europe [2,3].

Vancomycin is generally used for treating infections caused by vancomycin-susceptible E. faecium [4]. Previous studies have determined the appropriate target trough concentrations or area under the curve (AUC)/ minimum inhibitory concentration (MIC) ratios of vancomycin for treating methicillin-resistant Staphylococcus aureus (MRSA) and reported the association between the vancomycin trough concentration or AUC/ MIC ratio and vancomycin's therapeutic effect against MRSA bacteremia. Accordingly, a trough concentration of $10-20 \mu \mathrm{g} / \mathrm{mL}$ and an AUC/MIC $\geq 400$ was recommended in the guidelines for therapeutic drug monitoring of vancomycin $[5,6]$.

Further, a recent report showed that an AUC/MIC $\geq 389$ was associated with reduced mortality for various Enterococcus bacteremias [7]; however, few studies on the association between the vancomycin trough concentration or AUC/MIC ratio and the therapeutic effect against only $E$. faecium bacteremia have been conducted.

In this study, we investigated the associations between vancomycin pharmacokinetic/pharmacodynamic parameters, patient characteristics, and mortality in patients with E. faecium bacteremia.

\section{Methods}

\section{Study design and setting}

We performed a retrospective observational study. The study was conducted between April 1, 2012 and February 28, 2018 at a single 694-bed acute care hospital in Osaka, Japan.

\section{Participants and data collection}

The study included participants treated with vancomycin (VANCOMYCIN HYDROCHLORIDE for I.V. Infusion $0.5 \mathrm{~g}\lceil\mathrm{MEEK}\rfloor$, Meiji Seika Pharma Co., Ltd., Tokyo, Japan) for E. faecium bacteremia. Only the first episode of $E$. faecium bacteremia was considered. Patients were followed up until discharge or in-hospital death within 30 days after diagnosis. Patients with polymicrobial bacteremia were only included in the analysis if they had received antibiotics that were active in vitro against the other coinfection pathogens.

Vancomycin was prescribed on the basis of the hospital protocol (Table 1) by physicians.

The following patient data were extracted from medical records using predesigned forms: demographic characteristics (body weight, height, age, sex, underlying
Table 1 Protocol for vancomycin administration

\begin{tabular}{llll}
\hline $\begin{array}{l}\text { Creatinine clearance } \\
(\mathrm{mL} / \mathrm{min})\end{array}$ & Initial dose $(\mathrm{mg} / \mathrm{kg})$ & $\begin{array}{l}\text { Maintenance } \\
\text { dose } \\
(\mathrm{mg} / \mathrm{kg})\end{array}$ & Interval \\
\hline$\geq 70$ & $25-30$ (Loading dose) & $15-20$ & 8 or $12 \mathrm{~h}$ \\
$50-70$ & $10-15$ & $12 \mathrm{~h}$ \\
$30-49$ & $10-15$ & $24 \mathrm{~h}$ \\
$<30$ & $7.5-12.5$ & $24 \mathrm{~h}$ \\
\hline
\end{tabular}

diseases, and admission to the intensive care unit), diagnosis of infectious diseases and source(s) of infection(s), clinical laboratory data (alanine aminotransferase, alkaline phosphatase, serum creatinine, and total bilirubin levels; platelet and white blood cell counts), Glasgow Coma Scale, ratio of partial pressure of arterial oxygen to fraction of inspired oxygen $\left(\mathrm{PaO}_{2} / \mathrm{FiO}_{2}\right)$, urine output, 30-day all-cause mortality, administration dose of vancomycin, and initial trough concentration of vancomycin at the time of steady-state (before the fourth or more administration of vancomycin), as recommended by the guidelines [5, 6].

Patients were excluded from the study if they received renal replacement therapy (hemodialysis or continuous hemodiafiltration), had an unmeasured serum vancomycin concentration, had any unmeasured laboratory values as mentioned above, or received antibiotics other than vancomycin for the treatment of E. faecium bacteremia.

\section{Microbiology}

Species identification and susceptibility tests were performed in the clinical laboratory using an automated microdilution method (BD Phoenix ${ }^{\mathrm{mm}}$ Automated Microbiology System; Becton, Dickinson and Co., Tokyo, Japan). The pathogens were considered resistant to ampicillin or vancomycin if they exceeded the Clinical and Laboratory Standards Institute breakpoints (MIC of $\geq 16 \mu \mathrm{g} / \mathrm{mL}$ for ampicillin and $\geq 32 \mu \mathrm{g} / \mathrm{mL}$ for vancomycin).

\section{Definitions}

The onset of bacteremia was defined as the date of the collection of the first blood culture that yielded E. faecium. The suspected source of bacteremia was identified using information from the medical records.

The Charlson Comorbidity Index (CCI), Sequential Organ Failure Assessment (SOFA) score, augmented renal clearance (ARC) risk score, and estimated creatinine clearance (CLcr) were calculated as described previously [8-11]. CLcr was evaluated at the time of blood culture collection and the measurement of the initial trough concentration of vancomycin.

The SOFA score was calculated at the onset of E. faecium bacteremia and at the first measurement of the vancomycin 
trough concentration; furthermore, the difference between the two scores was calculated.

Acute kidney injury was evaluated using the Kidney Disease Improving Global Outcomes (KDIGO) criteria [12].

Treatment administered before the susceptibility results were obtained was defined as empirical therapy, whereas antimicrobial therapy given after the susceptibility data had become available was defined as definitive therapy. Treatment regimens were classified as monotherapy (treatment with 1 in vitro active agent) or combination therapy (treatment with $\geq 2$ in vitro active agents for isolated pathogens from blood culture).

The AUC from 0 to $24 \mathrm{~h}\left(\mathrm{AUC}_{24}\right)$ of vancomycin was calculated through the Bayesian approach using therapeutic drug monitoring software (Vancomycin MEEK TDM analysis software Ver 3.0; Meiji Seika Pharma Co., Ltd., Tokyo, Japan). In addition, the $\mathrm{AUC}_{24} / \mathrm{MIC}$ ratio was calculated using the $\mathrm{AUC}_{24}$ calculated for each case and the MIC obtained from microbiology test results. However, when the MIC was $\leq 0.5 \mu \mathrm{g} / \mathrm{mL}$, the $\mathrm{AUC}_{24} /$ MIC ratio was calculated with an MIC of $0.5 \mu \mathrm{g} / \mathrm{mL}$.

\section{Endpoints}

The primary endpoint of the study was the association between the vancomycin trough concentration or the $\mathrm{AUC}_{24} / \mathrm{MIC}$ ratio of $\leq 389$ and mortality in patients with E. faecium bacteremia. Secondary endpoints included the factors associated with all-cause mortality; these were compared between patients in the 30-day all-cause mortality and survival groups. Moreover, we performed stratified analyses by patients with acute kidney injury on pharmacokinetics of vancomycin and subgroup analysis of patients with a high CCI score ( $\geq 5$ points).

\section{Statistical analysis}

Data were analyzed using JMP ${ }^{\circ}$ 9.0.2 (SAS Institute Inc., Cary, NC, USA). Bivariate associations between the binary outcomes of 30-day all-cause mortality and patient characteristics were assessed using the Fisher's exact test for categorical variables and the Mann-Whitney $U$ test for continuous variables. All statistical analyses were conducted at a significance level of 0.05 .

\section{Results}

The patient characteristics are presented in Table 2 . Among 87 patients diagnosed with E. faecium bacteremia during the study period, 45 patients (27 [60.0\%] men and 18 [40.0\%] women) were analyzed (Fig. 1). A total of 12 of the 45 patients (26.7\%) died within 30 days after E. faecium bacteremia was diagnosed and were classified as the '30-day all-cause mortality group'. The 33 surviving patients were classified as the 'survival group.'
The median CCI was significantly different between the two groups (6 points [IQR, $5-7$ points] in the 30-day all-cause mortality group vs. 3 points [IQR, 2-6 points] in the survival group; $P=0.0081$ ). The median SOFA score at the onset of E. faecium bacteremia was not significantly different between the groups ( 3 points [IQR, 1-6 points] in the 30-day all-cause mortality group vs. 2 points [IQR, $0-3$ points] in the survival group; $P=$ 0.060). In contrast, the median SOFA score at the first measurement of the vancomycin trough concentration was significantly different between the groups ( 6 points [IQR, 4-8 points] in the 30-day all-cause mortality group vs. 2 points [IQR, $0-2$ points] in the survival group; $P<0.0001$ ).

The suspected origins of the infections are summarized in Table 2 . In the overall study population ( $n=45$ patients), the most common probable source of bacteremia was biliary tract infection in 22 patients (5 patients in the 30-day all-cause mortality group and 17 patients in the survival group); cases of endocarditis and meningitis were not included.

The pharmacokinetics and administration of vancomycin are shown in Table 3. There was a significant difference in the vancomycin trough concentration between the two groups $(20.5 \mu \mathrm{g} / \mathrm{mL}$ [IQR, 13.4$26.7 \mu \mathrm{g} / \mathrm{mL}]$ in the 30 -day all-cause mortality group vs. $14.6 \mu \mathrm{g} / \mathrm{mL}$ [IQR, $9.3-19.2 \mu \mathrm{g} / \mathrm{mL}$ ] in the survival group; $P=0.022$ ). However, we identified no significant differences in the number of patients with evaluated acute kidney injury (5 of 12 patients in the 30-day all-cause mortality group vs. 6 of 33 patients in the survival group; $P=0.11)$ and in the ARC risk score (1 point [IQR, $0-1$ points] in the 30-day all-cause mortality group vs. 1 point [IQR, $1-1$ points] in the survival group; $P=0.09$ ) between the groups (Table 2).

The $\mathrm{AUC}_{24}(721 \mu \mathrm{g} / \mathrm{mL} \cdot \mathrm{h}$ [IQR, $380-860 \mu \mathrm{g} / \mathrm{mL} \cdot \mathrm{h}]$ in the 30-day all-cause mortality group vs. $651 \mu \mathrm{g} / \mathrm{mL} \cdot \mathrm{h}$ [IQR, 462-952 $\mu \mathrm{g} / \mathrm{mL} \cdot \mathrm{h}$ ] in the survival group; $P=0.77$ ) and $\mathrm{AUC}_{24} / \mathrm{MIC}$ ratio (792 [IQR, 332-1040] in the 30-day all-cause mortality group vs. 707 [IQR, 450-1304] in the survival group; $P=0.76$ ) were not significantly different between the groups. Further, 3 of 12 patients $(25.0 \%)$ in the 30-day all-cause mortality group and 5 of 33 patients $(15.2 \%)$ in the survival group had a vancomycin $\mathrm{AUC}_{24} /$ MIC ratio of $\leq 389(P=0.66)$.

Stratified analyses by patients with acute kidney injury on pharmacokinetics of vancomycin are shown in Table 4. Regardless of the presence or absence of acute kidney injury, there was no significant difference between the 30-day all-cause mortality group and the survival group with respect to pharmacokinetics of vancomycin.

No significant difference was observed in the number of days from blood culture collection to vancomycin administration ( 1 day [1-4 days] in the 30-day all-cause 
Table 2 Patient and microbiological characteristics

\begin{tabular}{|c|c|c|c|c|}
\hline Characteristic & $\begin{array}{l}\text { All patients } \\
(N=45)\end{array}$ & $\begin{array}{l}\text { 30-day all-cause mortality group } \\
(N=12)\end{array}$ & $\begin{array}{l}\text { Survival group } \\
(N=33)\end{array}$ & $P$-value \\
\hline Sex (men: women) & $27: 18$ & $8: 4$ & $19: 14$ & $0.74^{i)}$ \\
\hline Age (years) ${ }^{a}$ & $73(64-83)$ & $71(63-79)$ & 75 (63-84) & $0.38^{\mathrm{ii})}$ \\
\hline Height $(\mathrm{cm})^{\mathrm{a}}$ & $158.0(151.8-166.0)$ & $159.2(153.5-169.4)$ & $156.7(150.3-165.3)$ & $0.24^{\mathrm{ii})}$ \\
\hline Body weight $(\mathrm{kg})^{\mathrm{a}}$ & $51.0(42.8-56.9)$ & $56.5(48.6-68.6)$ & $48.8(41.5-54.7)$ & $0.012^{\mathrm{ii})}$ \\
\hline Number of ICU admissions & $12(26.7 \%)$ & $3(25 \%)$ & $9(27.3 \%)$ & $0.11^{\mathrm{i})}$ \\
\hline ID specialist interventions & $27(60.0 \%)$ & $6(50.0 \%)$ & $21(63.6 \%)$ & $0.50^{i)}$ \\
\hline $\begin{array}{l}\text { Monotherapy } \\
\text { (number of patients) }\end{array}$ & 45 & 12 & 33 & $1.00^{i)}$ \\
\hline $\operatorname{ALT}(I U / L)^{a}$ & $30(16-55)$ & $25(13-44)$ & $33(16-64)$ & $0.29^{\text {ii) }}$ \\
\hline $\operatorname{ALP}(I \cup / L, N=40)^{a}$ & $544(302-1245)$ & $557(306-822, N=10)$ & $545(286-1467, N=30)$ & $0.53^{\text {ii) }}$ \\
\hline T-Bil $(\mathrm{mg} / \mathrm{dL})^{\mathrm{a}}$ & $0.9(0.5-3.1)$ & $1.0(0.5-2.8)$ & $0.8(0.5-3.3)$ & $0.63^{\mathrm{ii})}$ \\
\hline WBC $\left(\times 10^{3} / \mu \mathrm{L}\right)^{\mathrm{a}}$ & $8.5(4.9-12.3)$ & $12.5(7.0-23.8)$ & $7.4(4.8-11.3)$ & $0.047^{\text {ii) }}$ \\
\hline $\operatorname{PLT}\left(\times 10^{3} / \mu \mathrm{L}\right)^{\mathrm{a}}$ & $176.0(101.5-269.5)$ & $101.5(86.0-15)$ & $237.0(127.0-303.0)$ & $0.0056^{\mathrm{ii}}$ \\
\hline $\mathrm{SCr}(\mathrm{mg} / \mathrm{dL})^{\mathrm{a}}$ & $0.81(0.66-1.00)$ & $0.79(0.65-0.96)$ & $0.83(0.66-1.01)$ & $0.75^{\mathrm{ii})}$ \\
\hline $\mathrm{eGFR}\left(\mathrm{mL} / \mathrm{min} / 1.73 \mathrm{~m}^{2}\right)^{\mathrm{a}}$ & $67.0(52.0-78.0)$ & $71.0(51.3-78.0)$ & $66.0(52.0-77.0)$ & $0.69^{\mathrm{ii})}$ \\
\hline $\mathrm{CLCr}(\mathrm{mL} / \mathrm{min})^{\mathrm{ab}}$ & $53.6(39.4-78.1)$ & $61.7(51.4-89.1)$ & $48.9(36.7-73.4)$ & $0.10^{\mathrm{ii})}$ \\
\hline ARC risk score ${ }^{a}$ & $1(1-1)$ & $1(0-1)$ & $1(1-1)$ & $0.09^{\text {ii) }}$ \\
\hline Acute kidney injury patients ${ }^{c}$ & $11(24.4 \%)$ & $5(41.7 \%)$ & $6(18.2 \%)$ & $0.11^{\text {i) }}$ \\
\hline $\mathrm{CCl}^{\mathrm{a}}$ & $4(2-6)$ & $6(5-7)$ & $3(2-6)$ & $0.0081^{\text {ii) }}$ \\
\hline SOFA score $(a)^{a}$ & $2(0-4)$ & $3(1-6)$ & $2(0-3)$ & $0.060^{\mathrm{ii}}$ \\
\hline SOFA score $(b)^{a}$ & $2(0-5)$ & $6(4-8)$ & $2(0-2)$ & $<0.0001^{\text {ii) }}$ \\
\hline SOFA score (b)-(a) ${ }^{a}$ & $0(0-1)$ & $3(0-3)$ & $0(-1-0)$ & $<0.0001^{\text {ii) }}$ \\
\hline \multicolumn{5}{|l|}{ Suspected origin of infection } \\
\hline Catheter-related infection & 4 & 3 & 1 & \multirow[t]{5}{*}{ N.A } \\
\hline Biliary tract infection & 22 & 5 & 17 & \\
\hline Urinary tract infection & 5 & 2 & 3 & \\
\hline Intra-abdominal infection & 5 & 0 & 5 & \\
\hline Unknown & 9 & 2 & 7 & \\
\hline \multicolumn{5}{|c|}{ MIC of vancomycin for Enterococcus faecium } \\
\hline$\leq 0.5 \mu \mathrm{g} / \mathrm{mL}$ & 14 & $4(33.3 \%)$ & $10(30.3 \%)$ & \multirow[t]{4}{*}{ NA } \\
\hline $1.0 \mu \mathrm{g} / \mathrm{mL}$ & 29 & $7(58.3 \%)$ & $22(66.7 \%)$ & \\
\hline $2.0 \mu \mathrm{g} / \mathrm{mL}$ & 1 & 0 & $1(3.0 \%)$ & \\
\hline $4.0 \mu \mathrm{g} / \mathrm{mL}$ & 1 & $1(8.3 \%)$ & 0 & \\
\hline Positive polymicrobial blood culture & 15 & 3 & 12 & $0.72^{i)}$ \\
\hline
\end{tabular}

SOFA score (a): at the onset of E. faecium bacteremia

SOFA score (b): at the first measurement of the vancomycin trough concentration

$A L T$ alanine aminotransferase, $A L P$ alkaline phosphatase, $A R C$ augmented renal clearance, $C C l$ Charlson Comorbidity Index, $C L c r$ estimated creatinine clearance, eGFR estimated glomerular filtration rate, ICU intensive care unit, ID infectious disease, MIC minimum inhibitory concentration, NA not available, PLT platelet count, $\mathrm{SCr}$ serum creatinine level, SOFA Sequential Organ Failure Assessment, T-Bil total bilirubin, WBC white blood cell count

i) Fisher's exact test, ii) Mann-Whitney $U$ test

${ }^{a}$ Expressed as median (interquartile range, IQR)

${ }^{b}$ Calculated by the Cockcroft-Gault equation

'Patients with acute kidney injury evaluated at the first measurement of the vancomycin trough concentration

${ }^{\ddagger}$ The $P$-value was calculated by processing the comparison between the 30-day all-cause mortality group and the survival group by an appropriate statistical analysis 


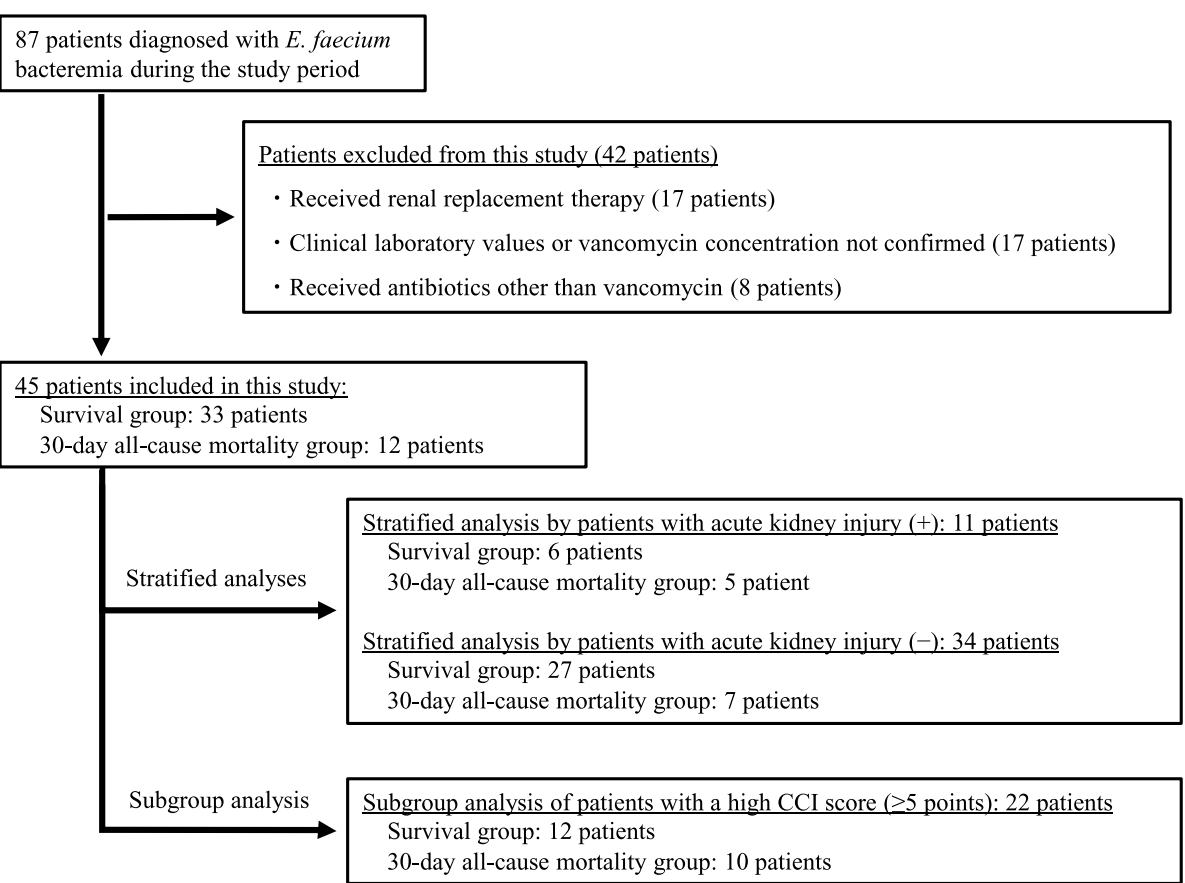

Fig. 1 Flowchart of patient selection. E. faecium, Enterococcus faecium; CCl, Charlson comorbidity index

mortality group vs. 3 days [1-4 days] in the survival group; $P=0.30$ ) between the two groups (Table 3 ).

The characteristics of E. faecium and positive polymicrobial blood culture results are summarized in Table 2. All 45 E. faecium cases were susceptible to vancomycin and resistant to ampicillin. Most strains had a vancomycin MIC value of $1 \mu \mathrm{g} / \mathrm{mL}$ (in 29 strains), followed by $\leq 0.5 \mu \mathrm{g} / \mathrm{mL}$ (in 14 strains). We observed no significant difference in polymicrobial bacteremia between the two groups (3 of 12 patients in the 30-day all-cause mortality group vs. 12 of 33 patients in the survival group; $P=0.72$ ).

The results of the subgroup analyses in patients with a high CCI score are depicted in Table 5. There was no significant difference in the CCI score $(6$ points [IQR, 6-8 points] in the 30-day all-cause mortality group vs. 6 points [IQR, $6-7$ points] in the survival group; $P=0.77$ ) and the median SOFA score at the onset of E. faecium bacteremia ( 2 points [IQR, 1-6 points] in the 30 -day all-cause mortality group vs. 1 point [IQR, 1-5 points]

Table 3 Pharmacokinetics and administration of vancomycin

\begin{tabular}{|c|c|c|c|c|}
\hline Characteristic & $\begin{array}{l}\text { All patients } \\
(N=45)\end{array}$ & $\begin{array}{l}\text { 30-day all-cause } \\
\text { mortality group } \\
(N=12)\end{array}$ & $\begin{array}{l}\text { Survival group } \\
(N=33)\end{array}$ & $P$-value ${ }^{\dagger}$ \\
\hline Vancomycin trough concentration $(\mu \mathrm{g} / \mathrm{mL})^{a}$ & $15(9.7-22.3)$ & $20.5(13.4-26.7)$ & 14.6 (9.3-19.2) & $0.022^{i)}$ \\
\hline $\begin{array}{l}\text { Frequency of vancomycin administration until the first measurement } \\
\text { of the vancomycin trough concentration }{ }^{\text {a }}\end{array}$ & $3(3-5)$ & $3(3-5)$ & $3(3-5)$ & $0.36^{\mathrm{i})}$ \\
\hline Vancomycin $\mathrm{AUC}_{24}(\mu \mathrm{g} / \mathrm{mL} \cdot \mathrm{h})^{\mathrm{a}}$ & 665 (444-898) & 721 (380-860) & 651 (462-952) & $0.77^{\mathrm{i})}$ \\
\hline Vancomycin $\mathrm{AUC}_{24} / \mathrm{MIC}$ ratio ${ }^{\mathrm{a}}$ & 725 (450-1186) & 792 (332-1040) & 707 (450-1304) & $0.76^{\mathrm{i})}$ \\
\hline Number of cases with failure to achieve a vancomycin $\mathrm{AUC}_{24} / \mathrm{MIC}$ ratio $\leq 389$ & $8(17.8 \%)$ & $3(25.0 \%)$ & $5(15.2 \%)$ & $0.66^{\mathrm{ii})}$ \\
\hline Duration of vancomycin administration (days) ${ }^{a}$ & $11.5(7.0-16.5)$ & $7(4.0-11)$ & $15(10-25)$ & $<0.001^{\mathrm{i})}$ \\
\hline End of vancomycin administration due to death & 8 & 8 & 0 & - \\
\hline Vancomycin used for empirical therapy & $29(64.4 \%)$ & $9(75.0 \%)$ & $20(60.6 \%)$ & $0.49^{\mathrm{ii})}$ \\
\hline Duration from blood culture collection to vancomycin administration (days) ${ }^{a}$ & $3(1-4)$ & $1(1-4)$ & $3(1-4)$ & $0.30^{\mathrm{i})}$ \\
\hline
\end{tabular}

$A \cup C_{24}$ estimated area under the curve from 0 to $24 \mathrm{~h}$, MIC minimum inhibitory concentration i) Mann-Whitney $U$ test, ii) Fisher's exact test

${ }^{\text {a }}$ Expressed as medians and interquartile ranges (IQR)

${ }^{\dagger}$ The $P$-value was calculated by processing the comparison between the 30 -day all-cause mortality group and the survival group by an appropriate statistical analysis 
Table 4 Stratified analyses by acute kidney injury on pharmacokinetics of vancomycin

\begin{tabular}{|c|c|c|c|c|}
\hline & Characteristic & 30-day all-cause mortality group & Survival group & $P$-value \\
\hline \multirow[t]{5}{*}{ Acute kidney injury (+) } & Number of patients & 5 & 6 & - \\
\hline & Vancomycin trough concentration $(\mu \mathrm{g} / \mathrm{mL})^{\mathrm{a}}$ & $26(22.3-33.2)$ & $12.8(9.1-30.5)$ & $0.20^{i)}$ \\
\hline & Vancomycin $\mathrm{AUC}_{24}(\mu \mathrm{g} / \mathrm{mL} \cdot \mathrm{h})^{\mathrm{a}}$ & $859(393-971)$ & $794(486-1052)$ & $0.86^{i)}$ \\
\hline & Vancomycin $\mathrm{AUC}_{24} / \mathrm{MIC}$ ratio $^{\mathrm{a}}$ & $859(427-971)$ & 809 (459-1409) & $0.86^{i)}$ \\
\hline & $\begin{array}{l}\text { Number of cases with failure to achieve a } \\
\text { vancomycin } \mathrm{AUC}_{24} / \mathrm{MIC} \text { ratio } \leq 389\end{array}$ & 1 & 0 & 0.45 ii) \\
\hline \multirow[t]{5}{*}{ Acute kidney injury (-) } & Number of patients & 7 & 27 & - \\
\hline & Vancomycin trough concentration $(\mu \mathrm{g} / \mathrm{mL})^{\mathrm{a}}$ & $15.0(12.7-21.4)$ & $14.6(9.3-18.2)$ & $0.30^{i)}$ \\
\hline & Vancomycin $\mathrm{AUC}_{24}(\mu \mathrm{g} / \mathrm{mL} \cdot \mathrm{h})^{\mathrm{a}}$ & $498(354-765)$ & $645(417-853)$ & $0.48^{i)}$ \\
\hline & Vancomycin $\mathrm{AUC}_{24} / \mathrm{MIC}$ ratio $^{\mathrm{a}}$ & $725(277-1531)$ & $707(417-1319)$ & $0.75^{\mathrm{i})}$ \\
\hline & $\begin{array}{l}\text { Number of cases with failure to achieve a } \\
\text { vancomycin } \mathrm{AUC}_{24} / \mathrm{MIC} \text { ratio } \leq 389\end{array}$ & 2 & 5 & $0.61^{\text {ii) }}$ \\
\hline
\end{tabular}

$A U C_{24}$ estimated area under the curve from 0 to $24 \mathrm{~h}, M / C$ minimum inhibitory concentration

i) Mann-Whitney $U$ test, ii) Fisher's exact test

${ }^{a}$ Expressed as medians and interquartile ranges (IQR)

${ }^{\dagger}$ The $P$-value was calculated by processing the comparison between the 30 -day all-cause mortality group and the survival group by an appropriate statistical analysis

in the survival group; $P=0.40$ ) between the groups. However, there was a significant difference in the vancomycin trough concentration between the two groups $(23.2 \mu \mathrm{g} / \mathrm{mL}$ [IQR, $14.4-28.3 \mu \mathrm{g} / \mathrm{mL}$ ] in the 30 -day all-cause mortality group vs. $10.5 \mu \mathrm{g} / \mathrm{mL}$ [IQR, 8.9$15.7 \mu \mathrm{g} / \mathrm{mL}$ ] in the survival group; $P=0.0069$ ) and the median SOFA score at the first measurement of the vancomycin trough concentration (6 points [IQR, 4-7 points] in the 30-day all-cause mortality group vs. 2 point [IQR, $0-2$ points] in the survival group; $P=0.014$ ).

\section{Discussion}

This study found no significant association in bacteremia cases caused by E. faecium, either with the $\mathrm{AUC}_{24} / \mathrm{MIC}$ ratio or the appropriate trough concentration of vancomycin, with mortality. Rather, the SOFA score during the measurement of the initial trough concentration of vancomycin was higher in the 30-day all-cause mortality group than in the survival group, suggesting that disease severity was associated with mortality.

The mortality rate of patients with E. faecium bacteremia has been reported as $25.0-34.6 \%$ [13-16], and is similar to the 30-day all-cause mortality rate in our study (26.7\%) that included patients with E. faecium bacteremia treated with vancomycin only.

Previous studies showed the following risk factors for mortality in patients with E. faecium bacteremia: current corticosteroid use, intensive care unit admission [13], a high CCI score, pulmonary infection, and nosocomial infection [14]. A recent retrospective study reported reduced mortality in patients with enterococcal (including E. faecium) bacteremia with a vancomycin $\mathrm{AUC} / \mathrm{MIC}_{\mathrm{E}-\text { test }}$ ratio $\geq 389$ [7]. However, in that study [7], vancomycin was administered according to hospital protocol, and the median blood concentration of vancomycin was lower than the blood concentration recommended for Staphylococcus bacteremia, in particular, in patients who died; thus, we hypothesize that the vancomycin dose was inadequate.

In our study, among all patients, the CCI was significantly higher in the 30-day all-cause mortality than in the survival group. However, in the subgroup analyses of patients with a high CCI score, prognostic factors at baseline such as the SOFA score and CCI were not significantly different between the 30-day all-cause mortality and survival groups. In our study, vancomycin was also administered according to hospital protocol; however, the median blood concentration of vancomycin in both groups achieved or exceeded the recommended concentration for the treatment of MRSA infection according to the guidelines for therapeutic drug monitoring of vancomycin $[5,6]$. Furthermore, the effect of the pharmacokinetics of vancomycin, such as the frequency of acute kidney injury and ARC risk score, were not significantly different between the groups. However, the median blood concentration of vancomycin was significantly higher in the 30-day all-cause mortality group than in the survival group in the total study population and in patients with a high CCI score. In contrast, the proportion of patients achieving $\mathrm{AUC}_{24} / \mathrm{MIC}$ ratios $\leq 389$ (for Enterococci) was not different between the two groups, both in the entire study population and in the subgroup of patients with a high CCI score. Therefore, our results suggest that the trough blood concentration and the $\mathrm{AUC}_{24} / \mathrm{MIC}$ ratio of vancomycin that were recommended for MRSA treatment were not associated with mortality in patients with E. faecium bacteremia. 
Table 5 Characteristics of patients with a high Charlson Comorbidity Index ( $\geq 5$ points) as well as pharmacokinetics and pharmacodynamics of vancomycin

\begin{tabular}{|c|c|c|c|c|}
\hline Characteristic & $\begin{array}{l}\text { All patients } \\
(N=22)\end{array}$ & $\begin{array}{l}\text { 30-day all-cause } \\
\text { mortality group } \\
(N=10)\end{array}$ & $\begin{array}{l}\text { Survival group } \\
(N=12)\end{array}$ & $P$-value ${ }^{\dagger}$ \\
\hline Body weight $(\mathrm{kg})^{\mathrm{a}}$ & $52.7(45.7-63.0)$ & $60.4(46.8-71.0)$ & $48.7(42.6-54.3)$ & $0.044^{i)}$ \\
\hline WBC $\left(\times 10^{3} / \mu \mathrm{L}\right)^{\mathrm{a}}$ & $9.1(4.0-13.2)$ & $13.2(7.4-17.4)$ & $5.8(3.5-11.4)$ & $0.048^{i)}$ \\
\hline $\operatorname{PLT}\left(\times 10^{3} / \mu \mathrm{L}\right)^{\mathrm{a}}$ & $154(97.5-206.5)$ & $102(76-152)$ & $181(125-292)$ & $0.021^{i)}$ \\
\hline $\mathrm{CCl}^{\mathrm{a}}$ & $6(6-7)$ & $6(6-8)$ & $6(6-7)$ & $0.77^{i)}$ \\
\hline SOFA score (a) & $2(1-5)$ & $2(1-6)$ & $1(1-5)$ & $0.40^{\mathrm{i})}$ \\
\hline SOFA score (b) & $3(1-6)$ & $6(4-7)$ & $2(0-2)$ & $0.014^{i)}$ \\
\hline Acute kidney injury patients" & $8(36.4 \%)$ & $4(40.0 \%)$ & $4(33.3 \%)$ & $1.00^{\mathrm{ii}}$ \\
\hline ARC risk score ${ }^{a}$ & $1(0-1)$ & $1(0-1)$ & $1(0-1)$ & $0.57^{\text {i) }}$ \\
\hline \multicolumn{5}{|l|}{ Pharmacokinetics and pharmacodynamics of vancomycin } \\
\hline Vancomycin trough concentration $(\mu \mathrm{g} / \mathrm{mL})^{a}$ & $14.8(9.9-25.3)$ & $23.2(14.4-28.3)$ & $10.5(8.9-15.7)$ & $0.0069^{\mathrm{i})}$ \\
\hline Vancomycin $\mathrm{AUC}_{24}(\mu \mathrm{g} / \mathrm{mL} \cdot h)^{\mathrm{a}}$ & $721(498-943)$ & $745(488-885)$ & $658(499-1065)$ & $1.00^{\text {i) }}$ \\
\hline Vancomycin $\mathrm{AUC}_{24} / \mathrm{MIC}$ ratio ${ }^{\mathrm{a}}$ & $826(561-1142)$ & $859(663-1194)$ & $730(515-1242)$ & $0.74^{i)}$ \\
\hline $\begin{array}{l}\text { Number of cases with failure to achieve a vancomycin } \\
\mathrm{AUC}_{24} / \mathrm{MIC} \text { ratio } \leq 389\end{array}$ & $10(45.5 \%)$ & $5(50.0 \%)$ & $5(41.7 \%)$ & $1.00^{\mathrm{ii}}$ \\
\hline \multicolumn{5}{|c|}{$\begin{array}{l}A U C_{24} \text { estimated area under the curve from } 0 \text { to } 24 \mathrm{~h}, C C I \text { Charlson Comorbidity Index, MIC minimum inhibitory concentration, PLT platelet count, SC } \\
\text { Organ Failure Assessment, WBC white blood cell count } \\
\text { i) Mann-Whitney U test, ii) Fisher's exact test } \\
\text { aExpressed in medians and interquartile ranges (IQR) } \\
{ }^{+} \text {The } P \text {-value was calculated by processing the comparison between the } 30 \text {-day all-cause mortality group and the survival group by an appropriate } \\
\text { statistical analysis } \\
\text { IPatients with acute kidney injury evaluated at the first measurement of the vancomycin trough concentration } \\
\text { SOFA score (a): at the onset of E. faecium bacteremia } \\
\text { SOFA score (b): at the first measurement of the vancomycin trough concentration }\end{array}$} \\
\hline
\end{tabular}

One of the reasons may be related to the antimicrobial activity of vancomycin against Gram-positive bacteria. Vancomycin has bactericidal activity against Gram-positive pathogens (including Staphylococci and Streptococci) and bacteriostatic activity against enterococci [17]. Generally, bacteriostatic antibiotics require phagocytic cells to definitely eliminate bacteria and are therefore considered less effective without an efficient immune response [18]. Accordingly, a previous study showed the importance of using bactericidal antibiotics in the treatment of endocarditis or meningitis [19]. Another study concluded that the categorization of antibiotics as bacteriostatic and bactericidal is likely not relevant in clinical practice if these antibiotics are used for abdominal infections, skin and soft tissue infections, and pneumonia [18]. In conclusion, the difference in the therapeutic effect on bacteremia between bactericidal and bacteriostatic antibiotics remains unclear. In our study, no cases of endocarditis and meningitis were included.

The cases in the 30-day all-cause mortality group were more severe illness than those of the survival group at the first measurement of vancomycin trough concentration, and thus, the patients were considered to have lower immunity. Therefore, we considered that a high serum concentration of vancomycin does not necessarily increase the drug's therapeutic effect against enterococcal infection.
In this study, we considered disease severity and patient characteristics as risk factors for mortality in patients with E. faecium bacteremia. In particular, patients in the 30-day all-cause mortality group had higher SOFA scores at the first measurement of the vancomycin trough concentration than those in the survival group, suggesting that the 30-day all-cause mortality group had higher disease severity.

Our study has some limitations. This was an observational study conducted at a single center, and the number of cases was limited. Unlike in a recent retrospective study [7], in our study, the MIC was measured using an automated microdilution method; moreover, a different calculation method of the $\mathrm{AUC}_{24}$, the Bayesian method that used one-point trough concentration and the population data of Staphylococcus aureus infections, was used. Therefore, the pharmacokinetics of acute kidney injury patients was not included in the population data, so AUC may be more inaccurate for these cases. Moreover, it is not possible simply to compare the AUC or AUC/MIC between our study and that of a recent retrospective study [7]. In both, in patients who died and those who survived, the blood concentration of vancomycin was within the effective treatment range for Staphylococcus aureus in many cases. As our study did not include enough cases with low $\mathrm{AUC}_{24} / \mathrm{MIC}$ ratios, 
we could not verify that a low $\mathrm{AUC}_{24} / \mathrm{MIC}$ ratio leads to a poor prognosis. In addition, in our case, CCI was higher in the 30-day all-cause mortality cases than in survival cases, and $\mathrm{CCI} \leq 5$ points patients in the 30 -day all-cause mortality cases group were very few. Therefore, we consider that there is a need for further study in cases with lower severity.

\section{Conclusions}

Our results suggest that the trough blood concentration and the $\mathrm{AUC}_{24} / \mathrm{MIC}$ ratio of vancomycin that are recommended for MRSA treatment were not associated with mortality in patients with E. faecium bacteremia. These conclusions are different from those of a recent retrospective study [7]. For this reason, further studies (in particular, prospective trials) are needed to clarify the association between the vancomycin trough concentration or the vancomycin AUC and mortality in patients with $E$. faecium bacteremia.

\section{Abbreviations}

ARC: Augmented renal clearance; AUC: Area under the curve; $\mathrm{AUC}_{24}$ : Estimated AUC from 0 to $24 \mathrm{~h}$; CCl: Charlson Comorbidity Index; CLcr: Estimated creatinine clearance; E. faecium: Enterococcus faecium; KDIGO: Kidney Disease Improving Global Outcomes; MIC: Minimum inhibitory concentration; MRSA: Methicillin-resistant Staphylococcus aureus; $\mathrm{PaO} 2 / \mathrm{FiO} 2$ : Ratio of partial pressure of arterial oxygen to fraction of inspired oxygen; SOFA: Sequential Organ Failure Assessment

\section{Acknowledgments}

We would like to thank Editage (http://www.editage.jp) for English language editing.

\section{Funding}

This study did not receive any specific grant from funding agencies in the public, commercial, or not-for-profit sectors.

\section{Availability of data and materials}

All data generated or analyzed during this study are included in this published article.

\section{Authors' contributions}

IN designed the study; performed the data collection, analysis, and interpretation; and wrote the initial draft of the manuscript. KS and KI contributed to the data analysis and interpretation as well as critically reviewed the manuscript. RS and KY critically reviewed the manuscript. All authors approved the final version of the manuscript and agree to be accountable for all aspects of the study by ensuring that questions related to the accuracy or integrity of any part of the study are appropriately investigated and resolved.

\section{Ethics approval and consent to participate}

This study was conducted in compliance with the standards of the Declaration of Helsinki and the current ethical guidelines. The study was approved by the ethics committee board of the Osaka National Hospital, reference number: 16148. To protect patient privacy, identifying data were encrypted in an electronic database. Written informed consent was exempted because no intervention was involved, and patient-identifying data were not included in the study.

\section{Consent for publication}

Not applicable.

\section{Competing interests}

The authors declare that they have no competing interests.

\section{Publisher's Note}

Springer Nature remains neutral with regard to jurisdictional claims in published maps and institutional affiliations.

Received: 21 January 2019 Accepted: 9 April 2019

Published online: 16 May 2019

\section{References}

1. Yuen GJ, Ausubel FM. Enterococcus infection biology: lessons from invertebrate host models. J Microbiol. 2014;52:200-10.

2. O'Driscoll T, Crank CW. Vancomycin-resistant enterococcal infections: epidemiology, clinical manifestations, and optimal management. Infect Drug Resist. 2015;24:217-30.

3. Japan Nosocomial Infections Surveillance. Annual Open Report 2015 (All Facilities): Japan Nosocomial Infections Surveillance (JANIS) (CLSI2012 Version) Clinical Laboratory Division. https://janis.mhlw.go.jp/english/report/ open_report/2015/4/1/ken_Open_Report_Eng_201500_clsi2012.pdf. Accessed 1 Dec 2018

4. Vazquez-Guillamet $\mathrm{C}$, Kollef MH. Treatment of gram-positive infections in critically ill patients. BMC Infect Dis. 2014;14:92

5. Rybak M, Lomaestro B, Rotschafer JC, et al. Therapeutic monitoring of vancomycin in adult patients: a consensus review of the American Society of Health-System Pharmacists, the Infectious Diseases Society of America, and the Society of Infectious Diseases Pharmacists. Am J Health Syst Pharm. 2009;66:82-98.

6. Matsumoto $\mathrm{K}$, Takesue $\mathrm{Y}$, Ohmagari $\mathrm{N}$, et al. Practice guidelines for therapeutic drug monitoring of vancomycin: a consensus review of the Japanese Society of Chemotherapy and the Japanese Society of Therapeutic Drug Monitoring. J Infect Chemother. 2013;19:365-80.

7. Jumah MTB, Vasoo S, Menon SR, De PP, Neely M, Teng CB. Pharmacokinetic/ pharmacodynamic determinants of vancomycin efficacy in enterococcal bacteremia. Antimicrob Agents Chemother. 2018;62:e01602-17.

8. Quan H, Li B, Couris CM, et al. Updating and validating the Charlson comorbidity index and score for risk adjustment in hospital discharge abstracts using data from 6 countries. Am J Epidemiol. 2011;173:676-82.

9. Vincent JL, Moreno R, Takala J, et al. The SOFA (sepsis-related organ failure assessment) score to describe organ dysfunction/failure. On behalf of the working group on sepsis-related problems of the European Society of Intensive Care Medicine. Intensive Care Med. 1996;22:707-10.

10. Udy AA, Roberts JA, Shorr AF, Boots RJ, Lipman J. Augmented renal clearance in septic and traumatized patients with normal plasma creatinine concentrations: identifying at-risk patients. Crit Care. 2013;17:R35.

11. Cockcroft DW, Gault MH. Prediction of creatinine clearance from serum creatinine. Nephron. 1976;16:31-41.

12. Khwaja A. KDIGO clinical practice guidelines for acute kidney injury. Nephron Clin Pract. 2012;120:c179-84.

13. Gudiol C, Ayats J, Camoez M, et al. Increase in bloodstream infection due to vancomycin-susceptible Enterococcus faecium in cancer patients: risk factors, molecular epidemiology and outcomes. PLoS One. 2013;8:e74734.

14. Billington EO, Phang SH, Gregson DB, et al. Incidence, risk factors, and outcomes for Enterococcus spp. blood stream infections: a population-based study. Int J Infect Dis. 2014;26:76-82.

15. Pinholt M, Ostergaard C, Arpi M, et al. Incidence, clinical characteristics and 30-day mortality of enterococcal bacteraemia in Denmark 2006-2009: a population-based cohort study. Clin Microbiol Infect. 2014;20:145-51.

16. Zhang Y, Du M, Chang Y, Chen LA, Zhang Q. Incidence, clinical characteristics, and outcomes of nosocomial Enterococcus spp. bloodstream infections in a tertiary-care hospital in Beijing, China: a four-year retrospective study. Antimicrob Resist Infect Control. 2017;6:73.

17. Cunha BA. Vancomycin revisited: a reappraisal of clinical use. Crit Care Clin. 2008:24:393-420.

18. Nemeth J, Oesch G, Kuster SP. Bacteriostatic versus bactericidal antibiotics for patients with serious bacterial infections: systematic review and metaanalysis. J Antimicrob Chemother. 2015;70:382-95.

19. Finberg RW, Moellering RC, Tally FP, et al. The importance of bactericidal drugs: future directions in infectious disease. Clin Infect Dis. 2004;39:1314-20. 